\title{
Guía de la revista 3 (1939-1941)
}

\author{
Miguel Angel Rodríguez Rea \\ Departamento Académico de Literatura
}

A David Sobrevilla

Fundada y dirigida por José Alfredo Hernández, Arturo Jiménez Borja y Luis Fabio Xammar, La revista 3 tiene gran significado en el desarrollo cultural del Perú contemporáneo. Su propósito de ser una experiencia cosmopolita a la vez que hurgar en las raíces nuestras, lo cumple con acierto y tallento. El rico y complejo bullir de las experiencias culturales que recoge en sus páginas muestra a un país que aspira a reflexionar sobre lo propio y lo contemporáneo como una sola forma de hallar una tradición de cultura viva y en constante renovación.

Su corta duración privó de ta consolídación de promesas y esbozos de creación originales. Queda el legado de un espiritu de búsqueda de caminos, propios para nuestra expresión cultural. Otras empresas como ésta dieron los frutos que aquí se pergeñaron (Letras Peruanas, Las Moradas, por citar las más conocidas). 3 estaba llamada a ser un

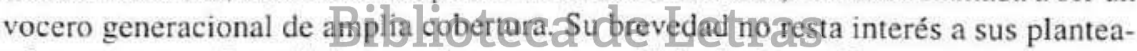
mientos y retos. Las ediciones pulcras, con agradable diseño gráfico, con textos expresamente solicitados, refrendaban una tarea trascendente en aras de una conciencia de lo nacional en la cultura.

La presente Guia ofrece el conjunto de las colaboraciones. Describe también los afamados Cuadernos de Cocodrilo (cuya estirpe retomaría Jorge Basadre para las ediciones de Historia*). Estos ejemplares de escasas y cuidadas páginas (a veces en papel couché). y sin numeración, acompañaban las tiradas de los números sueltos de la revista. El interés de los Cuadernos era recoger la producción de músicos, poetas y pintores con el fin de difundirlos. Al final, un Indice onomástico ayudará para consultar esta compilación.

* Cf. Miguel Angel Rodríguez Rea y Silvana Salazar, "Guía de la revista Historia (1943-1945)". Histórica. Vol. XIII. N². Diciembre de 1989. pp. 243-289. 


\section{N 1. JULIO DE 1939}

"Viñetas de Julio Camino Sánchez».

1. NúNEz, Estuardo

El sentimiento de la naturaleza en la nueva poesía del Perti.

Pp. 3-14.

2. VELARDE, HĖCTOR

Un loco en el año 3340.

Pp. 15-23.

Cuento humorístico.

3. Meireles, Cecilia

Cigarra.

P. [24].

Poesía.

4. Temple, Ella Dunbar

Curso de la literatura femenina a trayés del pertodo colonial en el Perti.

Pp. 25-56.

Contiene. La literatura devota. La literatura aristocrática. La literatura iluminada.

5. Devoto, Daniel J.

Selva.

P. [57].

Poesía.

\section{Biblioteca de Letras "Jorge Puccinelli Converso»}

6. Romero. Fernando

Santos Tarqui.

Pp. 58-65.

7. TAURo, Alberto

Retrato del general José Rufino Echenique.

Pp. 66-70.

Incluye una lámina, fuera de texto, con grabado de Echenique.

\section{NOTAS}

8. HeRnANDEZ, José Alfredo

Ricardo E. Molinari. Cinco canciones antiguas de amigo. Buenos Aires, Ediciones del Angel Galab. 1939, (Cuadernos del Cancionero de la Sirena, 1).

Pp. 71-72. 
9. Xammar. Luis Fabio

Agustín Zapata Golllán. La conquista criolla. Santa Fe, 1938.

Pp. $72-73$.

10. XAMmar. LUIS FABIO

Ernesto Pinto. Revelación de la imagen. Montevideo, 1938.

Pp. 73-74.

11. HeRnAiNdez, José Alfredo

Augusto Tamayo Vargas. Ingreso lírico a la poesía. Lima, Ediciones Palabra. 1939.

Pp. 74-75.

12. TAURo, Alberto

Jorge Basadre. Historia de la República del Perí. Lima, Librería e Imprenta Gil, 1939. Pp. 75-76.

13. HERNÁNDEZ. JOSÉ ALFREDO

Luis Cano. Nuevos romances camtares de la Colonia. Mercedes [Argentina], 1938.

P. 77.

14. Hernández, José Alfredo

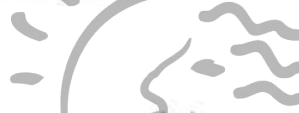

Carlos Alfonso Ríos. Temay manera de lo ausente. Pasamano, Talleres Unión, 1939.

P. 77.

15. s.f.

María Wiesse, La Tomíniea vidg de Mariano-Melgar. Lima, 1939.

P. 78.

16. s.f.

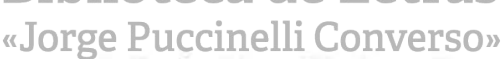

Esteban Pavletich. Leoncio Prado. Lima. Ediciones Demos, 1939.

Pp. 78-79.

17. s.f.

Teresa María Llona. Encrucijada. Lima. 1939.

P. 79.

18. s.f.

Amalia Cavero Mariátegui. Cantos de primavera. Lima, 1939.

P. 79.

\section{$\mathrm{N}^{\circ}$ 2. AGOSTO DE 1939}

“A Ricardo Peña, 1896-1939".

19. Miró Quesada S., Aurelio

Góngora encuentra al Inca Garcilaso.

Pp. 7-12. 
20. Negro, JuAN

Trance.

P. [13].

Poesía.

21. Arguedas, José María

Wáylluy:

Pp. 14-17.

Cuento.

22. PEÑa, Ricardo

Bandolero niño; comedia dramática en cuatro jornadas.

Pp. 18-49.

23. JimÉnez BorJa, ARTURo

Formas simples en el dibujo de los esquizofrénicos.

Pp. 50-61.

24. HERNÁNDEZ, JOSÉ ALFREDO

De la tortura.

P. [62].

Poesía.

25. Mejia BaCA, José

El nacimiento de "El Triste".

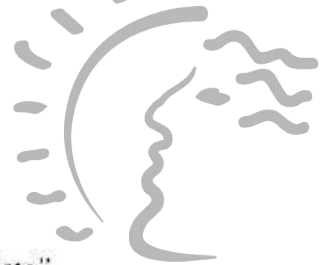

Pp. 63-68.

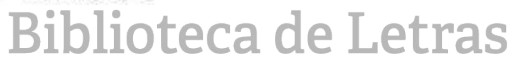

26. Martinez Hague, Cardor.ge Puccinelli Converso"

Palabras sobre Vallejo.

Pp. 69-70.

Necrología.

\section{NOTAS}

27. Carvajal, Luis de

Inquietud de angustia.

Pp. 71-77.

Sobre la poesía de Ricardo E. Molinari.

28. XAMMAR, LUIS FABIO

Arturo Marasso y la investigación literaria.

Pp. 78-79.

Comentario a su libro Cervantes y Virgilio (Buenos Aires, Instituto Cultural J.V. González, 1937). 
29. Jiménez Borja, Arturo

Rafael Jijena Sánchez y Bruno Jacobella. Las supersticiones. Buenos Aires, 1939. P. 80

30. JiMÉnEZ BorJa, ARTURo

Luis E. Valcárcel. Cuentos y leyendas inkas. Lima, 1939.

Pp. $80-81$.

31. HERNÁNDEZ, José ALFREdo

Juan Marín. Orestes y yo. Santiago de Chile, Editorial Nascimento, 1938.

P. 81.

32. NÚNEZ, EstuARDO

Moisés Sáenz. México íntegro. Carátula de José Sabogal. Lima. Imp. Torres Aguirre. 1939.

Pp. $81-82$.

33. JimÉnez BorJa. ARTURo

Francisco Izquierdo Ríos. Ande y Selva. 1939

Pp. 82-83.

34. Hernández, José Al.FRedo

Gicelda Zani. La cárcel del aire. Montevideo. Ed. Reuniones de Estudio, 1938.

P. 83 ,

35. Pereira, Raúl maria

Winett de Rokha. Cantoral. Santiago de Chile.

P. 84 .

"IJorge Puntiago de Chile.

\section{$N^{\circ}$ 3. DICIEMBRE DE 1939}

"Viñetas de Isajara".

36. BiRó de STERn, ANA

El estilo como elemento cultural.

Pp. 5-10.

Contiene. Estilo fisioplástico. 2. Estilo ideoplástico.

37. BASADRE, JoRgE

Diego de Peñalosa y Briceño.

Pp. 11-25. 
38. Peña, ENRIQUe

Canción.

P. [26].

Poesía.

39. Alcalde M.. Ricardo

El compadre Guisao.

Pp. 27-36.

Cuento.

40. Xammar, Luis Fabio

Lenguaje lírico de Cecília Meireles.

Pp. 37-46.

41. Valle, Rafael Heliodoro

Azul de Huejotzingo.

P. [47].

Poesía.

42. Benvenutto Murrieta, Pedro M

Trajes y tocados de las limeñas a través de euaum siglos.

Pp. 48-61.

43. Pereira, Raúl Maria

De lo gongórico en la poesía peruana actual.

Pp. 62-72.

Estudia este rasgo de estilo en Ricardo Peña, Martin Adán, Xavier Abril y José A.

Hernández. "Jorge Puccinelli Converso»

44. FingertT, MarCOS

Nieve ardiente.

P. [73].

Poesía.

45. Tamayo Vargas, Augusto

Aserradero.

Pp. 74-76.

Cuento.

46. PuccinelLi, Jorge

Abelardo Gamarra.

Pp. 77-80.

Reproducido en Turismo ( ${ }^{\circ}$ 96, Junio de 1944, p. [33]) con el título "Abelardo Gamarra, Pancho Fierro de nuestras letras".

[Entre las pp. 80 y 81 se incluye en hoja aparte el grabado Tomás de Agustín Zapata Gollán]. 


\section{COMENTARIOS}

47. NúNez. Estuardo

Poesia humana.

Pp. 81-83.

Reseña a: César Vallejo, Poemas humanos (1923-1938), París, Les Editions des Presses Modernes. 1939.

48. LEDGARD, RODOLFO

En torno al Ulises de Joyce.

Pp. 83-91.

\section{NOTAS}

49. TAuro, Alberto

Aida Cometta Manzoni. El mundo en la poesía de América española. Buenos Aires. 1939.

Pp. 92-93.

50. Hernández. José Alfredo

Zulma Núñez. Coplas de la soledad. Buenos Aires. Ed. Viau.

Pp. 93-94.

51. Hernández. José Alfredo

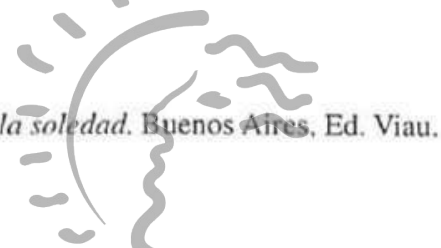

Alberto Wagner de Reyna. Rainer María Rilke (El poeta y el hombre). Lima, 1939. (Separata de la Revisja de la Uyiuersidad Católicg deh Perí).

Pp. 94-95.

52. XAMmar. LUIS FABIO

\section{«Jorge Puccinelli Converso»}

Juan Mantovani. La cultura, el arte y el Estado. Santa Fe, 1939.

Pp. 95-96.

53. Hernández. José Alfredo

Augusto Cambours Ocampo. Naufragio en la tierra. La Plata, Editorial "El Ateneo". 1939.

Pp. 96-97.

54. Xammar, LUIS Fabio

Revista Iberoamericana. Tomo I, N¹. Mayo de 1939.

Pp. 97-98.

55. HeRnÁNdez, José Al.rRedo

Luis de Paola. Canto para la muerte de Leopoldo Lugones. La Plata, 1939.

P. 99 . 
56. HeRnÁNdez José AlFredo

Horacio Correas. Poemas para la tierra de nadie. Rosario. 1939.

P. 99.

57. s.f.

Carlos Daniel Valcárcel. Garcilaso Inca, Lima, 1939.

P. 100 .

58. s.f.

Centenario del poeta coronado Luis Benjamín Cisneros. Buenos Aires, 1939.

P. 100.

\section{$\mathrm{N}^{\circ}$ 4. MARZO DE 1940}

59. Vélez Picasso, José M.

Un satírico olvidado: Mateo Rosas de Oquendo.

Pp. 5-15.

60. SchWAB, FEDERICO

Lo huachafo como fenómeno social.

Pp. 16-22.

61. Martin, Carlos

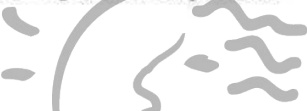

Compañera de nardo. blioteca de Letras
Pp. 23-24.

Poesía. "Jorge Puccinelli Converso»

62. SÁNCHEZ, LUIS ALbERTO

Bruno Traven (?) novelista desconocido.

Pp. 25-32.

Novelista norteamericano que residió, al parecer, en México, y con este seudónimo.

63. JimÉnEZ Borja, ARTURO

Primitivismo en la iconografia esquizofiénica.

Pp. 33-48.

"Trabajo realizado en el Servicio del Dr. Honorio Delgado, con material acumulado por él, desde hace 18 años".

64. Duncan, Elena

Para la muerte de Ricardo Peña.

P. [49].

Poesía. 
65. Champion, Emilio

Picardía de Caviedes.

Pp. 50-56.

"Fragmento de un Ensayo".

66. VÁSQUEZ, EMILIo

Exégesis de la poética de Alberto Guillén.

Pp. 57-67.

\section{NOTAS}

67. Anderson Imbert, EnriQue

Miniatura sobre Payró.

Pp. 68-69.

68. Peñaloza, Walter J.

La poesía de Pablo Neruda.

Pp. 69-87.

69. ROMERO, EMILIO

Lucio Mendieta y Núñez. La habitacion indígena, México, Instituto de Investigaciones Sociales de la Universidad Nacional Autónoma, 1939.

Pp. 88-89.

70. Hernández, José Alfredo

Aurelio Miró Quesada Sosa. Martín de Porres en el arte y en el folklore. Lima, 1940. P. 90 .

71. Xammar, LuIS FaBio

\section{"Jorge Puccinelli Converso»}

Jorge Guillermo Leguía. Estudios históricos. Santiago, 1939.

Pp. 90-91.

72. Carvajal, Luis de

Ediciones "Piedra y Cielo", Bogotá, 1939.

Pp. 91-94.

Reseñas a: Arturo Camacho Ramírez, Presagio de amor: Jorge Rojas, La ciudad sumergida; y, Carlos Martín, Territorio amoroso.

73. Hernández, José AlFredo

Héctor Velarde. El circo de Pitágoras. Lima, CIP, 1940.

P. 94.

74. Xammar, Luis Fabio

Samiento. La Plata, Universidad Nacional de la Plata, 1939.

P. 95. 
Volumen de homenaje que recoge trabajos de Rafael Alberto Arrieta, José A. Oría. Juan Casani, Ricardo Levene, Carlos Heras, Alberto Palcos, Antonio Salvadores, Fernando Márquez Miranda, Félix Aguilar. María Inés de Monner Sans y Emilio Azzarini.

75. TAURO, Alberto

María Luisa Vera. Poemas de niños tristes. México. 1939.

Pp. 95-96.

76. Xammar, Luis Fabio

Revista de Filologia Hispánica. Año I, N 1, Buenos Aires, 1939.

Pp. 96-97.

77. Xammar, LUIS Fabio

A. Waismann. La filosofía de Croce. Córdoba, 1939.

Pp. 97-98.

78. Tamayo Vargas, Augusto

Fernando Romero. Mar y playa Lima, Club del Libro Peruano, 1940.

Pp. 98-99.

79. Xammar, LUIS Fabio

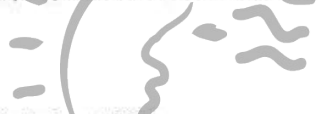

Azorín. Lobos Portos. Córdoba 1939

Pp. 99-100.

80. Buse de la Guerra, HermanN

Alejandro O. Deustua. La estética de fosé Vaśconcelos. Lima, Taller Gráfico de P. Barrantes, 1939. «Jorge Puccinelli Converso»

Pp. 100-102.

81. NúNẼEZ, Estuardo

Manuel González Prada. Baladas. Paris, Tip. de Louis Bellenand et fils, 1939.

Pp. 102-104.

82. s.f.

Cuadernos de la Asociación de Escritores Venezolanos. Caracas, Editorial "Elite".

P. 104.

83. s.f.

Viernes. $\mathrm{N}^{\circ}$ 6. Caracas, 1940.

P. 104.

El Secretario de Redacción de esta revista es el "fino poeta Pascual Venegas Filardo". 


\section{$\mathrm{N}^{\circ}$ 5. JUNIO DE 1940}

84. Meléndez, Concha

Recinto de Maria Zambrano.

Pp. 5-9.

85. JiMÉNEZ. JUAN RAMÓN

Vida.

P. [10].

Poesía.

86. Alegría, Ciro

La flauta de Pan.

Pp. 11-15.

"Primer capítulo de una novela inédita".

87. Pinto, ERnesto

Memoria de la florecida Navidad.

P. [16],

Poesía.

88. Tamayo Vargas, Augusto

Peri en trance de novela.

Pp. 17-34.

Sobre Mercedes Cabello de Carbonera.

Contiene. Trayectoria inicial y presentación intelectual. Crecimiento.

\section{Blolloteca ae uetras}

89. Hernández, José Alfrē̄olge Pulccinelli Converso»

Canción de fe a la Virgen Santa María.

Pp. [35-36].

Poesía.

90. Carvallo de Núñez, Cota y André Sas

Cantos infantiles. Melodia de Cota Carvallo de Núñez. Arreglos musicales de André Sas.

Pp. [37-43].

Acompaña a cada canción su respectiva partitura.

Contiene. El negro Pancho. La flor del Amancae; marinera. El río: huayno.

91. Tovar Velarde, Elias

$O$ 'Neill: su vida novelesca y su obra dramática.

Pp. 44-49.

Contiene. Punto de vista fundamental. El ansia viajera de $\mathrm{O}^{\prime}$ Neill. Técnica y estilo. 
NOTAS

92. Xammar. Luis Fabio

La fecha de nacimiento de Abraham Valdelomar:

Pp. 50-51.

93. Velarde G., Salvador

Anotación a Pirandello.

Pp. 51-60.

94. LEDGARD, RODOLFO

El poeta crucificado y la jauria.

Pp. 61-63.

Comentario al folleto del mismo título de Oscar Chávez (Santiago de Chile, Imp. de la Dirección General de Prisiones, 1940,57 p.) en el que trata de probar que Suramérica de Pablo de Rokha sirvió de base para que Joyce escribiera Finnegans Wake.

\section{BIBLIOGRAFIA}

95. ROMERO, EMILIO

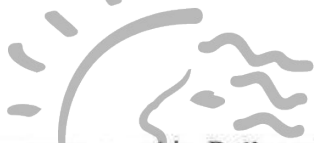

Vicente T. Mendoza. Romance y corrido. Prólogo de Jesús C. Romero. México, Ediciones de la Universidad Autónoma, 1939.

Pp. 64-65.

96. XAMMAR, LuIS FABIO

Rosa Arciniega. Play de vidas. Manizales, 1940.t1aS Pp. 65-66. «Jorge Puccinelli Converso»

97. JiméNez BorJa, Arturo

Tres libros colombianos, Ediciones Piedra y Cielo, Bogotá 1940.

Pp. 66-67.

Reseñas a los siguientes libros de poesía: Seis elegías y un himno de Eduardo Carranza; Regreso de la muerte de Tomás Vargas Osorio; y. El ángel desalado de Gerardo Valencia.

98. Xammar, Luis Fabio

Francisco Romero y Eugenio Pucciarelli. Lógica. Buenos Aires, 1939.

Pp. 67-68.

99. URTEAGa C.., FrankLin

España Peregrina. México, 1940.

Pp. 68-69

«Organo de la Junta de Cultura Española, cuyo presidente es José Bergamín, asesorado por José Carner, y Juan Larrea». 
100. Xammar, Luis Fabio

Luis A. Sánchez. América: novela sin novelistas. Santiago, 1940.

Pp. 69-70.

101. ROMERo, EMILIO

Ana Biró de Stern. Dibujo primitivo y dibujo infantil. Buenos Aires, 1938.

Pp. 70-71.

102. Xammar, Luis Fabio

Rufino José Cuervo. Disquisiciones filológicas. Bogotá, 1939.

P. 71.

103. HeRnÁNDEZ JosÉ AlFredo

Alto quién vive y santo y seña para 1940. Buenos Aires, Ed. Sol y Luna.

Pp. 71-72.

104. ROMERo, EMILIO

Revista Mexicana de Sociología. Año I. Vol. I. Nos. 4-5. 1939.

P. 72 .

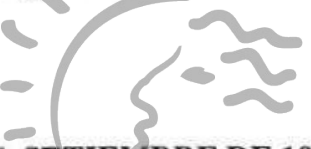

No 6. SETIEMBRE DE 1940

105. OROZ, RODOLFO

Reminiscencias virgilianas en Pedro de Oj̃a.

Pp. 5-11.

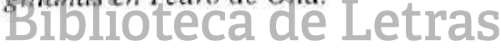

106. Diez-Canseco, José "Jorge Puccinelli Converso»

Romance de la luna zamba.

Pp. [12-13].

Poesía.

107. Martínez Hague, Carlos

El cuento nacional.

Pp. 14-25.

108. Paulotti, O.L. Y A. Dembo

La impresión de una visión a los últimos Mocoví.

Pp. 26-30.

«... los últimos Mocoví de la región de Napalpi (Chaco Argentino)".

109. MUelle, Jorge C.

Miraflores prehistórico.

Pp. 31-41.

Acompaña el artículo cuatro láminas de ceramios. 
110. Abril. Xavier

La rosa taurina (Sonetillo flamenco).

P. [42].

111. Xammar, Luis Fabio

El terremoto en la literatura peruana.

Pp. 43-56.

Reproducido en la Revista Iberoamericana (México, N¹5, Mayo 15 de 1944, pp. 107-120; véase la reseña. sin firma, a este artículo: La Prensa, Lima, 10 de setiembre de 1944 , p. 6).

112. Pareja Paz-Soldán, Carlos

Romance de la torre del oro.

Pp. [57-58].

Poesía.

113. TaUro, Alberto

José Pérez de Vargas, maestre y poeta.

Pp. 59-68.

114. Alarco, Luis Felipe

Búsqueda.

Pp. 69-73.

Relato.

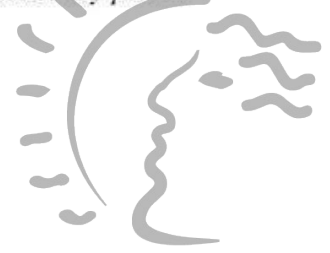

NOTAS

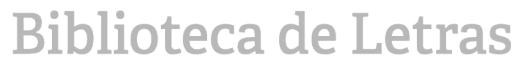

115. Hernández, José Aufrergoe Puccinelli Convel'so»

El lenguaje y el poeta.

Pp. 74-79.

Establece un paralelo entre Poeta en New York de Federico García Lorca (México, Editorial Séneca, 1940) y Poemas humanos de César Vallejo (París, 1939).

116. Carrillo Urdanivia, Graciela

El teatro de Federico García Lorca: Yerma y su obsesión de inmortalidad.

Pp. 79-81.

117. MaJluf, Emilio

Esquema geológico del Perú.

Pp. 81-86.

118. CaRvajal, LuIS DE

Ediciones, libros y editoriales.

Pp. 86-89.

Comenta sobre la producción de las casas editoras Losada y Sudamericana de Buenos Aires, y La Casa de España en México, y Editorial Séneca, (México). 


\section{BIBLIOGRAFIA}

119. Tamayo Vargas, Augusto

Juan Negro. Goces y muertes. Buenos Aires, Colección Continente, 1940.

Pp. 90-91.

120. Xammar. Luis Fabio

Aurelio Miró Quesada Sosa. Costa, Sierra y Montaña. Lima, 1940.

Pp. 91-92.

121. HERnÁNDEZ José AlFredo

El niño y su expresión. Santa Fe, Ministerio de Instrucción y Fomento.

Pp. 92-93.

122. TAmayo Vargas, Augusto

Dos ejemplares del Ministerio de Educación del Brasil.

Pp. 93-94.

J.G. de Magalhaes, Obras compleras. Vol. II. Suspiros poéticos e saudades. Edición anotada por Sousa de Silveira y Prefacio de Sergio Buarque de Holanda. Rio de Janeiro. 1940; Manuel Bandeira. Antologia des poetas brasileiros da fase parnasiana.

2a. ed. Rio de Janeiro, 1940 .

123. Xammar, LUIS FaBIO

José Jiménez Borja. Cien años de literatura y otros estudios críticos. Lima, 1940.

Pp. 94-95.

124. Hernández José Alpibiblioteca de Ietras

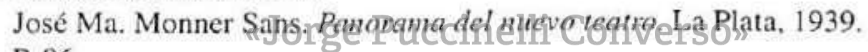

P. 96.

125. Xammar, LuIS Fablo

Paul Valéry. Cementerio marino. Traducción de R. Olivares Figuros. Caracas, 1940. Pp. 96-97.

126. Hernández, José Alfredo

3 libros mexicanos de Amigos Españoles de Fábula.

Pp. 97-98.

Cardenio, "monodrama» de Benjamín Jarnés; Son triste, libro de poesías de José

López Rubio; y, 7 poemas mexicanos de Eduardo de Ontañol.

127. BUSE de LA GUerra, HermanN

Mariano Iberico. El sentimiento de la vida cósnica. Lima 1939.

Pp. 98-100.

128. Hernández José Al.fredo

José Luis Sánchez Trincado. Pasión del arte nuevo. Caracas, 1940.

P. 100. 
129. Bernal, Dionicio Rodolfo

Antonio de Undurraga. Morada de España en Ultramar. Valparaíso, 1940.

Pp. 100-101.

130. Pereira, Raúl Marfa

Braulio Arenas. El mundo y su doble. Santiago. Ediciones, «Mandrágora», 1940.

Pp. 101-102.

131 s.f.

Letras. Segundo cuatrimestre de 1940.

P. 102.

132. s.f.

Pedagogía. Lima, junio de 1940.

Pp. 102-103.

133. s.f.

Mercurio Peruano. Lima, julio de 1940.

P. 103.

134, s.f.

Romance. Méjico, agosto de 1940.

P. 103.

135. s.f.

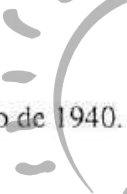

Revista de las Indias, Bogotá, junio de 1940

P. 104.

Biblioteca de Letras

136. NúNez, Estuardo "Jorge Puccinelli Converso"

Juan Bautista de Lavalle. Filosofía del Derecho y Docencia Jurídica. Lima, Librería e Imprenta Gil, 1939.

Pp. 104-105.

$N^{\circ}$ 7. DICIEMBRE DE 1940

[Incluye en separata, Indice. Años 1939-1940]

137. B ASADRE, JORGE

El montonero y la tapada.

Pp. 5-15.

138. Molinari, RiCARdo

Oda a una larga tristeza.

Pp. [16-17].

Poesía. 
139. Alcalde Mongrut, Ricardo

Ño Simón.

Pp. 18-26.

Relato.

140. LiRa, Miguel N.

Dos décimas de olvido.

P. [27].

Poesía.

141. Lohmann Villena, Guillermo

Apuntaciones sobre el arte dramático en Lima durante el Virreinato.

Pp. 28-57.

142. Venegas Filardo, Pascual

Canción a tu silencio.

P. [58].

Poesía.

143. SÁNCHEZ, LUIS ALBERTO

Una glosa sobre la cultura perhana.

Pp. 59-62.

144. Zevallos QuiÑones, Jorge

Un romance español del siglo XVIII en el Perú.

Pp. 63-70.

«... versos de trova hatiados en eldepartamento de ta Libertad...".

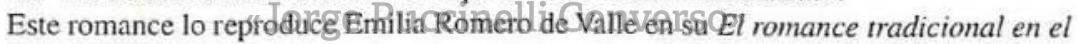
Perú, México, El Colegio de México, 1952, p. 71.

145. Peralta, Alejandro

El hombre del charango.

Pp. [71-72].

Poesía.

146. Velarde, Samuel

Morir:

Pp. 73-81.

Cuento.

\section{NOTAS}

147. Xammar, Luis Fabio

La Exposición del Libro Argentino.

Pp. 82-83. 
148. LEDGARD, RODOLFO

Rilke y la religiosidad en la poesía contemporánea.

Pp. 84-87.

149. Oyanedez Encinas, Arturo

Tendencias nuevas en la novela hispanoamericana.

Pp. 87-90.

\section{BIBLIOGRAFIA}

150. RoMERo, FERnANDO

Eduardo Martin Pastor. La vieja casa de Pizarro. Lima. 1940.

Pp. 91-92.

151. Xammar, Luis Fabio

John Gassner. Un decenio del drama americano. Washington, 1940.

Pp. 92-93.

152. Hernández, José Alfredo

Antonio Aita. Analecta. Buenos Aires, Editorial Pecse, 1940.

Pp. 93-94.

153. Peña, EnRIQue

Juan Guzmán Cruchaga. Avennura (Poemas). San Salvador, 1940.

Pp. 94-95.

154. XaMmar, LUIS FABIO

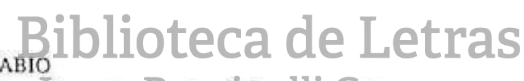

Enrique Barboza. Lapsicologia dien atista. Dima 4940 "

Pp. 95-96.

155. Hernández, José Alfredo

Otto d'Sola. Antología de la moderna poesía venezolana. Caracas, Biblioteca Venezolana de Cultura, 1940.

Pp. 96-97.

156. Xammar, LUIS FABIO

Augusto Tamayo Vargas. Perú en trance de novela. Lima 1940.

Pp. 97-98.

157. HernÁNdez, José Alfredo

Aurelio Miró Quesada Sosa. Artes y oficios del Perú. Lima, Editorial Lumen, 1940.

Pp. 98-99.

158. Hernández, José Alfredo

Vicente Gerbasi. Bosque doliente. Caracas 1940.

Pp. 99-100. 
159. HERNANDEZ JOSÉ ALFREDO

Rainer María Rilke. Poemas de la pobreza y de la muerte. La Plata, Hipocampo.

Pp. 100-101.

160. Xammar, Luis Fabio

Universidad. Santa Fe, junio de 1940.

P. 101 .

161 s.f.

Revista del Mar del Pacífico. Quito, noviembre de 1940.

P. 102.

162 s.f.

Garcilaso. Lima, noviembre de 1940.

p. 102.

N 8. MARZO-JUNIODE 1941

163. Miró Quesada Sosa, Aurelio

El Inca Garcilaso y una connedia de Tirso de Molina.

Pp. 5-13.

Amazonas en las Indias.

Reimpreso: Lima, Teatro de San Marcos, 1970, [8] + [2] p. (Serie IV, Estudios de Teatro Peruano, 81). Texto̊ mimeografiado.

Contiene. Oportunidad de 1 a composición. Huelfas det fmea Garcilaso. Gloria y fama del Inca. "Jorge Puccinelli Converso»

164. GUILLÉn, JoRGE

La vida real.

Pp. 14-[17].

Poesía.

165. Temple, Ella Dunbar

Títeres y titereros en la Lima de fines del siglo XVIII.

Pp. 18-30.

166. NÚNEZ, EstuARdo

Apunte sobre Thorton Wilder:

Pp. 31-35.

167. GarRIDO, JosÉ EULOGIO

El Ande.

Pp. 36-39.

Relato. 
168. Valle, Rafael Heliodoro

Contigo.

P. [40].

Poesía.

169. Alvarado Sánchez, José

Una rosa para Rimbaud.

Pp. 41-56.

170. Peña, EnRIQue

Ausencia de Ricardo.

P. [57].

Poesía.

Ricardo Peña.

171. CaRvajaL, LuIS DE

El grito contra el viento.

Pp. 58-66.

Cuento.

172. Tauro, Alberto

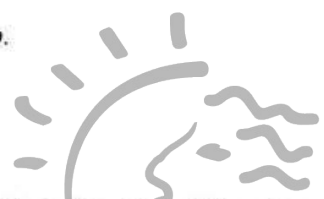

Presencia y definición del indigenismo literario.

Pp. 67-83.

También en Memoria del Segundo Congreso Internacional de Catedráticos de Literatura Iberoamericana, Los Angeles, 1940.

Se reproduce un fragmentoen Actas y trabajos científices del XXVIII/4 Congreso Intemacional de Americanistas (Lima, 1939), Lima, Librería e Imprenta Gil, 1942, t. II. pp. [147]-150. "Jorge Puccinelli Converso»

Contiene. El indigenismo, ante la evolución de la conciencia nacional. Atisbos indigenistas, a través de la evolución de la literatura peruana. Indigenismo: síntoma de resurgimiento colectivo. Indigenismo: manifestación intelectual de un fenómeno social concreto. El indigenismo como «reivindicación de lo autóctono». Originalidad y valor estético del indigenismo. El indigenismo, ante la crítica. Conclusiones.

173. LEDGARD, RoDOLFo

La realidad de Franz Kafka.

Pp. 84-91.

174. Rios, Luis AlberTo

Revolución.

Pp. 92-97.

Relato. 


\section{NOTAS}

175. Xammar. LuIS Fabio

Palabras de presentación pronunciadas en la Facultad de Letras de la Universidad Nacional Mayor de San Marcos, al iniciarse el ciclo de conferencias de don Ramón Pérez de Ayala.

Pp. 98-100.

176. HeRNANdez JosÉ Al.rRedo

Charla sustentada en el Instituto Cultural Peruano-Norteamericano.

Pp. 101-105.

Sobre su viaje a Norteamérica.

177. Carvajal, LUIS DE

Presencia y ausencia de la nostalgia.

Pp. 105-111.

Relato.

178. GUILLÉN, JoRGE

Carta a Héctor Velarde.

P. 111 .

Dirigida desde Wellesley. Massachusetts, con fecha 14 de enero de 1941, comenta entre otras cosas la aparición de su libro El circo de Pitágoras.

\section{BIBLIOGRAFIA Biblioteca de Letras}

179. Xammar. Luis Fabio "Jorge Puccinelli Converso »

Rodolfo Oroz. «El Vasauro», poema heroico de Pedro de Oña. Santiago, Prensas de la Universidad de Chile, 1941.

Pp. 112-113.

180. Hernández José Alfredo

Concha Meléndez. Entrada en el Perú. La Habana. «La Verónica», 1941.

Pp. 113-114.

181. Jiménez Borja. Arturo

María Rosa Macedo C. Ranchos de caña. Lima. 1941

P. 114 .

182. Xammar. Luis Fabio

José María Arguedas. Yawar fiesta. Lima, 1941.

Pp. 115-116. 
183. HeRnÁNDEZ, José Alfredo

Mario Florián. Tono de fauna. Cajamarca, 1941.

P. 116.

184. Xammar, LUIS FABIO

José Luis Bustamante y Rivero. Una visión del Perí. Montevideo, 1941.

Pp. 116-117.

185. Hernández, JosÉ Alfredo

Eduardo Caballero Calderón. Tipacoque. Bogotá, Talleres Gráficos «Mundo al Día». 1940.

Pp. 117-118.

186. Xammar, LUIS Fabio

Pedro Grases. Don Luis Correa, suma de generosidad en las letras venezolanas. Caracas, 1941.

Pp. 118-119.

187. Ríos, LuIS AlberTo

Macedonio Fernández. Una novela que Comienza.Santiago de Chile Ediciones Ercilla, 1941.

Pp. 119-121.

188. Xammar, Luis Fabio

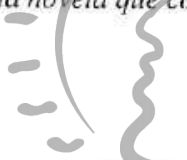

Gilberto González Contreras. Rubén Romero, el hombre que supo ver. La Habana, 1940.

Pp. 121-122. Biblioteca de Ietras

189. Olivas, Antonio

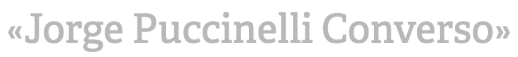

Fermín Peraza Sarausa. Anuario Bibliográfico Cubano, 1940. La Habana, Imprenta y Papelería "Alfa», 1941.

Pp. 123-124.

190. Buse de la Guerra, Hermann

Salvador de Madariaga. Vida del muy magnífico señor don Cristóbal Colón. Buenos Aires. Editorial Sudamericana, 1940.

Pp. 124-125.

191. Lugo P., Manuel

Joaquín Xirau. La filosofia de Husserl. Buenos Aires, 1941.

Pp. $125-126$.

192. Carvajal, Luis de

Cuadernos de Poesía. Barcelona-Madrid. Ediciones Patria.

P. 127. 
Dirigida por Jesús Nieto. El No 3 incluye una antología de poetas peruanos. En números anteriores esta revista dedicó sendos homenajes a Unamuno. Príncipe de Esquilache y Gutierre de Cetina.

193. Carvajal, Luis de

Manuel Maples Arce. Antología de la poesia mexicana moderna. Roma, 1940.

Pp. 127-128.

194. Olivas. Antonio

Luis Lama. Arriba! (Lecturas peruanas). Lima, 1941.

Pp. 128-129.

195. Hernández, José Alfredo

La Editorial Espasa-Calpe S.A. Buenos Aires.

P. 129.

Noticia de los títulos recientemente publicados: Doña Bárbara de Rómulo Gallegos; Lazarillo de Tormes; La pata de la raposa de Ramón Pérez de Ayala: Plenitud de Amado Nervo: Taras Bulba de Nicolís Gogol: y. Luis Candelas de Antonio Espina.

196. s.f.

Cultura Peruana. No2. Lima, 1941.

Pp. 129-130.

197. s.f.

Garcilaso. Año 2. No 3. Abril de 1941. Lima.

Pp. 130-131. Biblioteca de Letras

198. s.f. "Jorge Puccinelli Converso"

Alcor: (Sintesis bimensual de la Vida Americana). Buenos Aires, 1941.

P. 131 .

\section{No 9. SETIEMBRE-DICIEMBRE DE 1941}

«Viñetas de Alicia Bustamante».

[Incluye en separata, Indice. Año 1941]

199. MishKIN. BeRNARD

Posesión de la tierra en una aldea andina.

Pp. [5]-11.

En la comunidad de Kauri, en el Cuzco.

200. Diez-Canseco. José

José Joaquín de Larriva, clérigo.

Pp. 12-28. 
201. Velarde, HÉctor

Arquitectura del Renacimiento.

Pp. 29-41.

202. Xammar, LUIS Fabio

Virgilio y Juan de Arona.

Pp. 42-47.

203. González, Carlos Alberto

Parábola.

P. [48].

Poesía.

204. ARrósPide de LA Flor, CÉsAR

Un concertista del siglo pasado en Lima.

Pp. 49-55.

Luis Moreau Gottschalk, pianista norteamericano que visitó Lima en 1865.

205. Miró Quesada, Francisco

La absurdidad del mundo.

Pp. 56-64.

206. Mejia BaCA, JosÉ

Relato en el tambo.

Pp, 65-70.

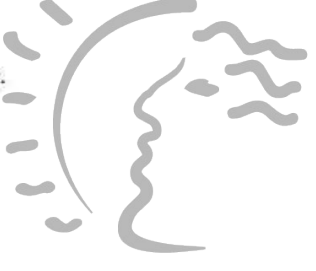

"Capítulo de la novela Sambambér.a de Ietras

207. Delgado, Amadeo "Jorge Puccinelli Convelso»

Poema.

Pp. [71-72].

208. Peñaloza R., Walter

Significado de don Ricardo Palma en nuestra cultura.

Pp. 72-95.

209. Mejia Xesspe, M.T.

Contribución al estudio de la geofagia en el Perí.

Pp. 96-102.

Contiene. I. Arcillas. II. Sustancias cálcicas. Bibliografía.

\section{NOTAS}

210. Hernández, José Alfredo

Viejas leyendas del Perú (Conferencia dictada en la Universidad Nacional Mayor de San Marcos).

Pp. $103-110$. 
211. Xammar, Luis Fabio

Teatro de Ricardo Peña Barrenechea (Prólogo a la audición de Bandolero Niño interpretado por la Asociación de Artistas Aficionados).

Pp. $110-112$.

212. SANCHEZ, LUIS AlBerto

Panorama hacia el alba: testimonio y clave.

Pp. 112-115.

Comentario a la novela de José Ferrando (Lima, Talleres Gráficos de la Editorial «El Universals, 1941).

Incluido en la segunda sección de su artículo «Dos notas sobre literatura peruana contemporánea» (Revista lberoamericana, México, Vol. IV, № 8, Febrero 15 de 1941, pp. 315-322).

213. FUENTES IBÁNEZ, M.F.

Alrededor de El mundo es ancho y ajeno, novela sudamericana.

Pp. $115-118$.

214. Deustua, Raúl.

La poesía de José María Eguref.

Pp. 119-128.

215. HernándeZ José Alfredo

Murthia de Osuna en la Universidad de San Marcos.

P. 128.

Poetisa española. Biblioteca de Letras

\section{BIBLIOGRaFia "Jorge Puccinelli Converso"}

216. XAMmar, LUIS FABIO

Amado Alonso. Poesía y estilo de Pablo Neruda. Buenos Aires, 1940.

Pp. 129-130.

217. Hernández Josê Al.fredo

Daniel J. Devoto. El arquero y las torres. La salamandra. Buenos Aires, Gulab y Aldabahor, 1940.

Pp. 130-131.

218. Xammar, Luis Fabio

Alberto Ulloa. Posición internacional del Perí. Lima, 1941.

Pp. 131-133.

219. Hernández, José Al.redeo

Leopoldo Lugones. Antología poética. Selección y prólogo de Carlos Obligado. Buenos Aires, Espasa-Calpe Argentina.

Pp. 133-134. 
220. Xammar, LUIS FABIO

Horacio Rega Molina. Oda provincial. Buenos Aires, 1940.

Pp. 134-136.

221. Hernández, José Alfredo

Estuardo Núñez. La prosa literaria del Perí en los últimos veinte años. Los Angeles.

California. Sobretiro de la Memoria del Segundo Congreso Internacional de Catedráticos de Literatura Iberoamericana

Pp. 136-137.

222. Xammar, LuIS Fabio

Obras completas de Luis Benjanin Cisneros mandadas publicar por el Gobiesno del Perí.

Pp. 137-138.

223. HernÁNdez, José Alfredo

Paul Marcoy. Viajes por los valles de la quina. Buenos Aire, Espasa-Calpe Argentina.

P. 138.

224. Ríos, Luis Alberto

Vicente Huidobro. El ciudadano del olvido. Santiago de Chile, Ediciones Ercilla.

1941.

Pp. $138-143$.

225. Pereira, Raúl María

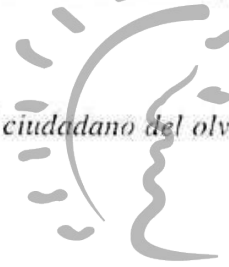

Rafael Heliodoro Valle Indice dela poesía cenwoanericana. Santiago de Chile, Editorial Ercilla, 1941 .

Pp. 143-144. "Jorge Puccinelli Converso»

226. Hernández, José Alfredo

D. W. Lawrence. La serpiente emplumada. Buenos Aires, Editorial Losada.

Pp. 144-145.

227. Hernández, José Alfredo

Elena Duncan. Las vivas llagas. La Plata, Cuadernos Viador, 1941.

P. 145 .

228. XAMMAR, LUIS FABIO

Alberto Jochamowitz. Baca Flor, hombre singular: Lima, 1941.

P. 146.

229. Pereira. Raúl Maria

Pascual Venegas Filardo. Música y eco de tu ausencia. Caracas, Ediciones Viernes, 1941.

P. 147. 
230. Olivas. Antonio

Marion Evelyn Wright. Juanito, el frejolito mexicano saltarín. Lima, 1941.

Pp. 147-148.

231. Cultura Pertuana. $N^{\circ} 4$. Setiembre de 1941.

P. 148.

232. s.f.

Letras. $\mathrm{N}^{\circ} 19$. Segundo cuatrimestre de 1941.

P. 148 .

233. s.f.

Mercurio Peruano. № 174. Setiembre de 1941.

Pp. 148-149.

234. s.f.

Peruanidad. $\mathrm{N}^{\circ} 1$, Noviembre de 1941.

P. 149.

235. Peña, Ricardo

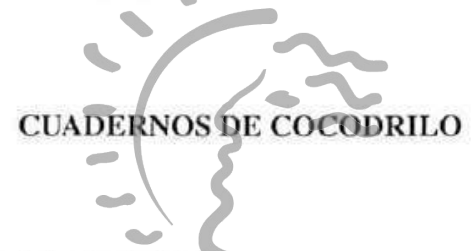

Cántico lineal. [8] p. [Anejo del $\mathrm{N}^{\circ}$ I]

236. Máscaras de baile. Coleccion de Arturo Jiménez Borja. [12] p. [Anejo del $\left.\mathrm{N}^{\circ} 1\right]$.

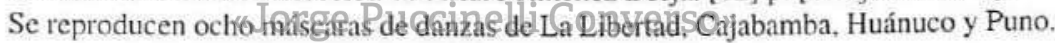

237. MARTIN ADÁN

La rosa de la espinela. [16] p. [Anejo del $\left.\mathrm{N}^{\circ} 2\right]$.

238. La pintura de Ricardo Peña. [8] p. [Anejo del $N^{\circ} 2$ ].

Incluye cinco reproducciones (Auto retrato, Cartón abstracto, Pasillo de negritos. Figura del mar: China de Chachapoyas).

239. Sánchez Málaga, Carlos

Dos corales a capella. [12] p. [Anejo del $\mathrm{N}^{\circ} 3$ ].

Incluye las partituras de Marinera: canción popular y Pajarillo errante.

240. VICENTE AZAR

Nueva canción de otoño. [16] p. [Anejo del $\mathrm{N}^{\circ} 3$ ].

241. Cueto Fernandini, Carlos

Poemas dispares. [12] p. [Anejo del $N^{\circ} 4$ ]. 
242. Instrumentistas y bailarines (Departamento de Puno). [16] p. [Anejo del No 4].

Texto de presentación por Arturo Jiménez Borja: pp. [11-14].

Incluye ocho fotografías.

243. Coreografia colonial. Acuarelas mandadas hacer por D. Baltasar Jaime

Compañón y Bujanda. S. XVIII. [16] p. [Anejo del No 4].

"Coreografía nacional", por Arturo Jiménez Borja: pp. [11-14].

Se reproducen ocho acuarelas.

244. Rodriguez César Atahualpa

Poemas. [8] p. [Anejo $\mathrm{N}^{\circ}$ 6].

245. Mercado, Guillermo

Canto a Sachaca. [8] p. [Anejo del $N^{\circ} 5$ ].

246. Mate peruano. Colecciones: Musco Nacional de Arqueología y Arturo Jiménez Borja. [20] p. [Anejo del $\left.\mathrm{N}^{\circ} 6\right]$.

"Mate peruano", por Arturo Jiménez Borja: pp. [16-19].

"Dibujos de Alejandro Gonzales"

"Fotos de Abraham Guillén".

247. Música popular. Este cuaderno sale bajo los cuidados de Carlos Sanchez

Málaga. [8] p. [Anejo del No 7]

Se incluyen cuatro partituras de las composiciones recopiladas por Leonardo Cerrón en Tarma.

248. FlorLÁN. MARIO

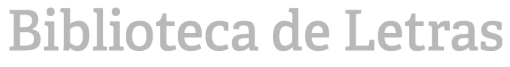

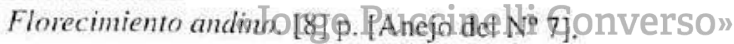

249. HARTH-TERRÉ, EMILIO

Arquitectura popular peruana. Fotografias del autor: [26] p.

[Anejo del $\mathrm{N}^{\circ} 8$ ].

"[La arquitectura popular]", por Emilio Harth-terrré: pp. [19-25].

Incluye 26 fotografías.

250. Leyendas del Peni. Recogidas por Artum Jiménez Borja. [8] p. [Anejo del No 8].

"Dibujos de Julia Codesido".

251. Ríos, Carlos Alfonso

Celebración del destino. [8] p. [Anejo del N8].

252. HernÁNDEZ, José Alfredo

El ángel agitado. [12] p. [Anejo del $\mathrm{N}^{\circ}$ 9].

«Este cuaderno lleva tres dibujos de esquizofrénicos, de la colección del Dr. Honorio Delgadom. 
253. Taquies según Guamán Pona. [20] p. [Anejo del ํㅜㄹ.

"Con la colaboración de J.M.B. Farfán".

"[Taquies]", por Arturo Jiménez Borja: pp. [13-19].

254. Sánchez Málaga. Carlos

Dos Lieder: Versos de Luis Fabio Xamunar. [16] p. [Anejo del No 9].

Los versos corresponden al libro Wayno.

\section{INDICE ONOMASTICO}

Abril. Xavier 43,110

Aguilar. Félix 74

Aita. Antonio 152

Alarco, Luis Felipe 114

Alcalde Mongrut. Ricardo 39,139

Alegría, Ciro 86, 213

Alonso. Amado 216

Alvarado Sánchez. José 169, 240

Anderson Imbert, Enrique 67

Arciniega, Rosa 96

Arenas. Braulio 130

Arguedas, José María 21, 182

Arrieta, Rafael Alberto 74

Arróspide de la Flor, César 204

Azorin 79

Biblio

Azzarini. Emilio 74

Baca-Flor, Carlos 228

Bandeira. Manuel 122

Bandeira Filho, Manuel Carneiro de Sousa, v.

Bandeira. Manuel

Barboza. Enrique 154

Barrantes Castro. Pedro 6, 80

Basadre. Jorge 12, 37, 137

Bellenand, Louis 81

Benvenutto Murrieta. Pedro Manuel $\mathbf{4 2}$

Bergamín. José 99

Bernal, Dionicio Rodolfo 129

Biró de Stern. Ana 36, 101

Buarque de Holanda, Sergio 122

Buse de la Guerra. Hermann 80, 127, 190

Bustamante y Rivero. José Luis 184

Caballero Calderón. Eduardo 185

Cabello de Carbonera. Mercedes 88
Camacho Ramírez, Arturo 72

Cambours Ocampo, Augusto 53

Cané, Luis 13

Carner, José 99

Carvajal, Luis de 27, 72, 118, 171, 177, 192, 193

Carvallo de Núñez, Cota 90

Carranza. Eduardo 97

Carrillo Urdanivia, Graciela 116

Casani, Juan 74

Cavero Mariátegui, Amalia 18

Caviedes, Juan del Valle 65

Cerrón, Leonardo 247

Cervantes Saavedra, Miguel de 28

Cetin- Gutierre ge 192

Champion, Emilio 65

Cháves, Oscar 94

Cisneros. Luis Benjamín 58, 222

Codesido, Julia $\mathbf{2 5 0}$

Colón, Cristóbal 190

Cometta Manzoni, Aída 49

Correa, Luis 186

Correas. Horacio 56

Croce. Benedetto 77

Cuervo, Rufino José 102

Cueto Fernandini, Carlos 241

Delgado, Amadeo 207

Delgado, Honorio 63, 252

Dembo, A. 108

Deustua. Alejandro O. 80

Deustua, Raúl 214

Devoto, Daniel J. 5, 217

Diez-Canseco. José 106, 200

Duncan, Elena 64, 227 
Echenique, José Rufino 7

Eguren, José María 214

Espina. Antonio 195

Esquilache. Francisco de Borja y Aragón,

Príncipe de 192

Farfán Ayerbe. José María Benigno 253

Fernández. Macedonio 187

Ferrando, José 212

Fierro. Pancho 46

Fingerit, Marcos 44

Florián, Mario 183, 248

Fuentes Ibáñez, Moisés F. 213

Gallegos. Rómulo 195

Gamarra, Abelardo M. 46

García Lorca, Federico 115, 116

Garcilaso de la Vega. Inca 57, 163

Garrido. José Eulogio 167

Gassner, John 151

Gerbasi. Vicente 158

Gogol, Nikolai Vasilieich 195

Gonzales, Alejandro 246

González, Carlos Alberto 203

González, J.V. 28

González Contreras. Gilberto 188

González Prada, ManueP \&

Grases. Pedro 186 "Jorge PuccinelliLonntresesaría 17

Guaman Poma de Ayala, Felipe 253

Guillén. Abraham 246

Guillén. Alberto 66

Guillén. Jorge 164, 178

Guzmán Cruchaga, Juan 153

Harth-terré, Emilio 249

Heras, Carlos 74

Hernández. José Alfredo 8, 11, 13, 14, 24,

$31,34,43,50,51,53,55,56,70,73,89$,

$103,115,121,124,126,128,152,155$,

$157-159,176,180,183,185,195,210,215$,

$217,219,221,223,226,227,252$

Huidobro. Vicente 224

Hussserl. Edmund 191

Iberico, Mariano 127

Izquierdo Ríos. Francisco 33

Lugones, Leopoldo 155, 219
Jacovella. Bruno 29

Jarnés. Benjamin 126

Jijena Sánchez, Rafael 29

Jiménez, Juan Ramón 85

Jiménez Borja, Arturo 23, 29, 30, 33, 63,

$97,181,246,236,242,243,246,250,253$

Jiménez Borja, José 123

Jochamowitz. Alberto 228

Joyce, James 48.94

Juan de Arona 202

Kafka. Franz 173

Lama. Luis 194

Larrea, Juan 99

Larriva. José Joaquín de 200

Lavalle. Juan Bautista de 136

Lawrence. David Herbert 226

Ledgard, Rodolfo 48, 94, 148, 173

Leguia.Jorge Guillermo 71

Levene, Ricardo 74

Lira, Miguel N. 140

Lohmann Villena, Guillermo 141

López Rubio, José 126

Lugo P., Manuel 191
Macedo C., María Rosa 181

Madariaga, Salvador de 190

Magalhaes, Domingo José Gonçalves de, v.

Magalhaes, José Gonçalves de

Magalhaes, José Gonçalves de 122

Majluf, Emilio 117

Mantovani, Juan 52

Maples Arce, Manuel 193

Marasso, Arturo 28

Marcoy, Paul [seud. de Laurent Saint-

Cricq] 223

Marín, Juan 31

Márquez Miranda. Fernando 74

Martin, Carlos 61, 72

Martín Adán 43. 237

Martín de Porres, san 70

Martin Pastor, Eduardo 150 
Martínez Compañón y Bujanda, Jaime 243

Martínez Hague, Carlos 26, 107

Meireles. Cecília 3, 40

Mejía Baca. José 25, 206

Mejía Xesspe. M. Toribio 209

Meléndez. Concha 84, 180

Melgar, Mariano 15

Mendieta y Núnez. Lucio 69

Mendoza. Vicente T. 95

Mercado. Guillermo 245

Miró-Quesada Cantuarias. Francisco 205

Miró-Quesada Sosa, Aurelio 19. 70. 120.

157. 163

Mishkin. Bernard 199

Molinari, Ricardo E. 8. 27, 138

Monner Sans. José María 124

Monner Sans, María Inés de 174

Moreau Gottschalk, Luis 204

Muelle. Jorge C. 109

Negro, Juan 20, 119

Neruda, Pablo 68. 216

Nervo. Amado 195

Nieto, Jesús 192

Núñez. Estuardo 1, 32, 47, 81, 136, 166,

Núñez. Zulma 50

Obligado. Carlos 219

Olivares Figueroa, R. 125

Olivas. Antonio 189, 194, 230

O'Neill. Eugene 91

Ontañol. Eduardo de 126

Oña. Pedro de 105, 179

Oría. José A. 74

Oroz, Rodolfo 105. 179

Osuna. Myrthia de 215

Oyanedes Encinas, Arturo 149

Palcos. Alberto 74

Palma. Ricardo 208

Paola. Luis de 55

Pareja Paz-Soldán, Carlos 112

Pastor, Eduardo Martín. v. Martín Pastor.

Eduardo

Paulotti. O.L. 108

Biblioteca Rokhar Pahlo de 94

Pavletich, Esteban 16

Payró. Roberto J. 67

Peña Barrenechea. Enrique 38, 153, 170

Peña Barrenechea, Ricardo 22, 43, 170 ,

$211,235,238$

Peñalosa y Briceño, Diego de 37

Peñaloza Ramella, Walter J. 68, 208

Peralta. Alejandro 145

Peraza Sarausa. Fermín 189

Pereira, Raúl María 35, 43, 130, 225, 229

Pérez de Ayala, Ramón 175, 195

Pérez de Vargas, José 113

Pinto, Ernesto 10,87

Pirandello, Luigi 93

Pizarro, Francisco 150

Pucciarelli. Eugenio 98

Puccinelli, Jorge 46

Rega Molina. Horacio 220

Rilke, Rainer María 51, 148, 159

Rimbaud, Arthur 169

Ríos. Carlos Alfonso 14, 251

Ríos, Luis Alberto 174, 187, 224

Rodríguez, César Atahualpa $\mathbf{2 4 4}$

Rojas, Jorge 72

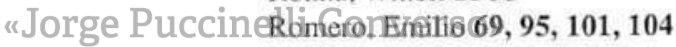

Romero. Fernando 6, 78, 150

Romero. Francisco 98

Romero, Jesús C. 195

Romero, Rubén 188

Romero de Valle, Emilia 144

Rosas de Oquendo, Mateo 59

Sabogal, José 32

Sáenz. Moisés 32

Salvadores, Antonio 74

Sánchez, Luis Alberto 62, 100, 143, 212

Sánchez Málaga. Carlos 239, 247, 254

Sánchez Trincado, José Luis 128

Sas, André 90

Schwab, Federico 60

Silveira. Sousa da 122

Sola, Otto d' 159 
Tamayo Vargas. Augusto $11, \mathbf{4 5}, \mathbf{7 8}, \mathbf{8 8}$, 119, 122. 156

Tauro. Alberto 7, 12, 49, 75, 113, 172

Téllez, Fray Gabriel, $v$. Tirso de Molina

Temple. Ella Dunbar 4, 165

Tirso de Molina 163

Tovar Velarde. Elías 91

Ulloa, Alberto 218

Unamuno, Miguel de 192

Undurraga, Antonio de 129

Urteaga C., Franklin 99

Valcárcel. Carlos Daniel 57

Valcárcel, Luis E. 30

Valdelomar, Abraham 92

Valencia, Gerardo 97

Valéry. Paul 125

Valle. Rafael Heliodoro 41, 168, 225

Vallejo, César 26, 47, 115

Vargas Osorio, Tomás 97

Vasconcelos. José 80

Vásquez. Emilio 66

Velarde, Héctor 2, 73, 178, 201
Velarde. Samuel 2, 73, 146, 178

Velarde G., Salvador $\mathbf{9 3}$

Vélez Picasso, José M. 59

Venegas Filardo, Pascual 83, 142. 229

Vera, María Luisa 75

Vicente Azar, $v$. Alvarado Sánchez. José

Virgilio Marón. Publio 28, 105. 202

Wagner de Reyna, Alberto 51

Waismann, A. 77

Wiesse, María 15

Wilder, Thorton 166

Wright, Mario evelyn 230

Xammar, Luis Fabio 9, 10, 28, 40, 52, 54, $71,74,76,77,79,92,96,98,100,102$, $111,120,123,125,147,151,154,156$, $160,175,179,182,184,186,188,202$, $211,216,218,220,222,228,254$

Xirau. Joaquín 191

Zani, Gicelda 34

Zapata Gollán, Agustín 9

Zevallos Quiñones, Jorge 144 